\title{
Douce tentation, planète rouge ou dieu de la guerre?
}

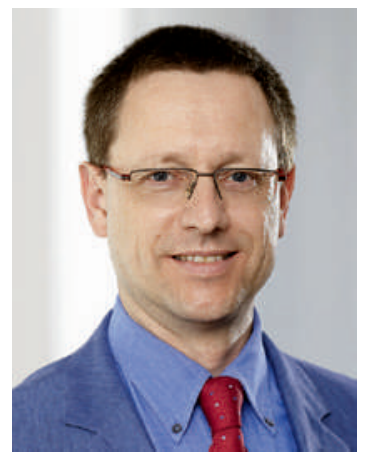

Involontairement, Mars évoque des images différentes en chacun de nous. Cependant, personne - ou presque - n'associe ce terme à un projet de la Confédération dédié à la saisie des données sur les soins médicaux dans notre pays. Après une présentation à la dernière Chambre médicale par les responsables des Offices fédéraux concernés, c'est à votre tour, chers confrères et chères consœurs, de prendre connaissance du projet MARS «Modules Ambulatoires sur les Relevés de la Santé» dans ce numéro du Bulletin des médecins suisses.

\section{La FMH s'attache à ce que le temps investi par les médecins soumis à l'obligation légale de transmettre leurs données soit réduit autant que possible.}

Se voir attribuer chaque jour toujours plus de tâches administratives nous exaspère autant que les collectes de données soulèvent des soupçons chez nous - rien d'étonnant au vu de l'utilisation hasardeuse qui peut en découler. Le politique s'est cependant appuyé sur une base légale pour donner des mandats clairs qui sont actuellement en cours de mise en œuvre. La FMH suit donc ces questions de près depuis déjà pas mal de temps et nous avons réussi à établir un dialogue constructif avec les responsables de l'Office fédéral de la statistique (OFS). Cette collaboration a pour objectif de répondre à la préoccupation de la FMH, soit s'assurer de la qualité de la formulation des questions en tenant compte aussi du point de vue médical afin de recueillir des réponses pertinentes. De par leur expérience pratique, les médecins savent qu'une bonne indication suivie d'une intervention réalisée dans les règles de l'art aboutit à un bon résultat mais ils savent également qu'une mauvaise indication ne donnera pas de bon ré- sultat malgré une intervention parfaite. L'interaction entre les questions et les réponses possibles obéit à la même logique.

\section{Pour recueillir des réponses pertinentes, un bon questionnaire doit tenir compte du point de vue des médecins.}

Le temps investi par les médecins soumis à l'obligation légale de transmettre leurs données doit être réduit autant que possible. Avec ses partenaires, la FMH offre la possibilité à ses membres d'autoriser d'un seul clic le transfert des données déjà collectées relatives à leur activité médicale. A cet égard, nous veillons à ce que toute l'attention requise soit portée sur les aspects de la protection des données - aussi bien dans l'intérêt de nos patients que de celui de nos membres. L'Office fédéral de la santé publique (OFSP) est donc appelé à élaborer une ordonnance conforme à la protection des données.

Si les données statistiques conduisent à des conclusions qualitatives trop hâtives reposant sur de simples coïncidences, il s'agit de reconnaître les limites de leur pertinence et d'éviter les doublons. Mais le cadre du mandat politique doit également être respecté: il s'agit d'éviter que l'exigence légitime de collecter des données dans un intérêt commun de solidarité soit détournée pour recueillir simultanément des données privées.

Dans ce numéro du BMS (page 859), vous trouverez un article détaillé de l'OFS présentant le projet. Vous pouvez également consulter toutes les informations importantes à ce sujet sur le site de la FMH (www.fmh.ch $\rightarrow$ Politique $\&$ thèmes $\rightarrow$ Démographie médicale $\rightarrow$ MARS).

Le projet Mars mobilise; la FMH continuera de s'engager de manière constructive et critique, et de vous proposer, chers membres, son soutien également à ce sujet.

Dr Christoph Bosshard, membre du Comité central de la FMH, responsable du département Données, démographie et qualité 